\title{
A Model for Neutrinos
}

\author{
B.G. Sidharth \\ G.P. Birla Observatory \& Astronomical Research Centre \\ B.M. Birla Science Centre, Adarsh Nagar, Hyderabad - 500063 (India)
}

\begin{abstract}
Neutrinos are the weirdest of elementary particles, nearly massless and chiral. They oscillate between flavours, furthermore. We try to find a rationale for this maverick behaviour.
\end{abstract}

\section{Introduction}

In 1930 Wolfgang Pauli regretted introducing the concept of the neutrino on the grounds that it would not be observable. However, thereafter as is well known, it was indeed observed in the context of weak interactions, even though it has continued to remain nature's most enigmatic particle. What is known is that its mass could be $\sim 10^{-8}$ the electron mass, even though what has actually been observed todate is the mass differences between different flavours of neutrinos. These particles are fermions which travel with nearly the speed of light. There are some $10^{90}$ of them in the universe and more recently a huge cosmic background of neutrinos has been observed [1]. Unlike usual fermions, the neutrino is described by the two component Weyl equation and thus exhibits chirality, one of its weird characteristics. John Wheeler has stressed that unless General Relativity can describe a neutrino, in particular, its unification with Quantum Theory would not be possible [2] Very recently the Gran Sasso Lab in Italy has reported that what started up at LHC, Geneva as a beam of $\mu$ neutrinos had switched flavours to appear as $\tau$ neutrinos at Gran Sasso. Could such effects answer the problem of deficit of solar neutrinos, is a question that comes up. 


\section{Two Dimensionality}

We now observe that the neutrinos are in some sense two dimensional entities and this would explain their maverick features. Firstly, the Dirac equation in two space dimensions can represent the neutrino as can be easily verified. Indeed this was pointed out by the author nearly twenty years back [3, 4] and more recently this again came to be of interest because of the work on graphene, which loosely is a two dimensional sheet of graphite. Here we come across the phenomena of quasi particles with distinctly neutrino like properties [5, 6, 7].

Specifically, we first linearize the relativistic energy momentum relation,

$$
E^{2}=p^{2} c^{2}+m_{0}^{2} c
$$

and write down the corresponding quantum mechanical equation as

$$
H \psi=\left[c \vec{\alpha} \cdot \vec{p}+\beta m_{0} c^{2}\right] \psi
$$

where

$$
\vec{p} \equiv \frac{\hbar}{\imath}\left(\frac{\partial}{\partial x} \frac{\partial}{\partial y}\right) \text { and } H \equiv \frac{\hbar}{\imath} \frac{\partial}{\partial t}
$$

Multiplying (2) by $H$ on the left side and $\left(c \vec{\alpha} \cdot \vec{p}+\beta m_{0} c^{2}\right)$ on the right side and comparing with equation (1) we get,

$$
\left(\alpha^{\imath} \alpha^{j}+\alpha^{j} \alpha^{\imath}\right)=2 \delta^{\imath j}, \alpha^{\imath} \beta+\beta \alpha^{\imath}=0, \beta^{2}=1, \imath j=1,2
$$

Equation (3) is satisfied if the set $(\vec{\alpha}, \beta)$ is the set of Pauli matrices.

Thus equation (21) represents a two component Fermion. Relativistic covariance of this equation can be easily verified. The neutrino is such a two component Fermion [8]. It is also well known that the reason the neutrino which satisfies the Dirac equation, or its special case, the Weyl equation has only two components is that its rest mass $m_{0}$ vanishes.

So if a Fermion can somehow be confined to two dimensions, for example to the surface of a thin strip of negligible thickness, for example graphene, then it should behave effectively like the massless and parity-violating neutrino. 
We must also remember that the cosmic background neutrinos are at the temperature $T \approx 3^{\circ} \mathrm{K}$ that is this collection is ultra cold and as such nearly mono energetic. We can argue that this leads to a bosonic behaviour as follows. Our starting point is the well known formula for the occupation number of a Fermion gas $[9]$

$$
\bar{n}_{p}=\frac{1}{z^{-1} e^{b E_{p}}+1}
$$

where, $z^{\prime} \equiv \frac{\lambda^{3}}{v} \equiv \mu z \approx z$ because, here, as can be easily shown $\mu \approx 1$,

$$
\begin{gathered}
v=\frac{V}{N}, \lambda=\sqrt{\frac{2 \pi \hbar^{2}}{m / b}} \\
b \equiv\left(\frac{1}{K T}\right), \text { and } \sum \bar{n}_{p}=N
\end{gathered}
$$

Let us consider in particular a collection of Fermions which is somehow made nearly mono-energetic, that is, given by the distribution,

$$
n_{p}^{\prime}=\delta\left(p-p_{0}\right) \bar{n}_{p}
$$

where $\bar{n}_{p}$ is given by (4).

This is not possible in general - here we consider a special situation of a collection of nearly mono-energetic particles in equilibrium which is the case with the Cosmic Background neutrinos.

By the usual formulation we have,

$$
N=\frac{V}{\hbar^{3}} \int d \vec{p} n_{p}^{\prime}=\frac{V}{\hbar^{3}} \int \delta\left(p-p_{0}\right) 4 \pi p^{2} \bar{n}_{p} d p=\frac{4 \pi V}{\hbar^{3}} p_{0}^{2} \frac{1}{z^{-1} e^{\theta}+1}
$$

where $\theta \equiv b E_{p_{0}}$.

It must be noted that in (7) there is a loss of dimension in momentum space, due to the $\delta$ function in (6) - in fact such a fractal two dimensional situation would in the relativistic case lead us to anomalous behaviour described elsewhere [10]. One way of looking at this is that dimensionality itself is connected to the virial distribution of velocities and in this special situation, there is hardly such a velocity spread. 
Indeed this two dimensionality aspect has entered physics through the holographic principle in the most recent Quantum Gravity approaches [11. Here to put it simply three dimensionality is perceptional as in the three dimensional image of a hologram. All the detail is contained in the two dimensional image. In the case of Black Holes, all information content is confined to its surface. Indeed the universe itself can be characterized as a Black Hole [12].

Furthermore in 1996 it was argued by the author (and A.D. Popova) that the universe is asymptotically two dimensional. A quick way to see this would be by observing the dynamics of the flat rotation curves of galaxies [13. Essentially, observations reveal, that for galaxies at large distances $R$, their mass goes as

$$
M \propto R^{n}, \quad \text { where } n \approx 2
$$

indicating two dimensionality.

All this suggests that the neutrino can be treated in the above context as a two dimensional object obeying the two dimensional Dirac or Weyl equation.

There is yet another way of looking at all this. It is well known, in the theory of the Dirac equation, that if we construct wave packets of positive energy solutions alone (or negative energy solutions alone) [14], we get

$$
\psi^{(+)}(x, t)=\int \frac{d^{3} p}{(2 \pi \hbar)^{1 / 2}} \sqrt{\frac{m c^{2}}{E}} \sum_{ \pm s} b(p, s) u(p, s) e^{-\imath p_{\mu} x \mu / \hbar}
$$

The expectation of the velocity operator is given by

$$
\mathbf{J}^{(+)}=\int \psi^{(+) \dagger} \operatorname{con} \psi^{(+)} \mathbf{d}^{\mathbf{3}} \mathbf{x}
$$

which leads to

$$
\mathbf{J}^{(+)}=\langle\mathbf{c} \alpha\rangle_{+}=\left\langle\frac{\mathbf{c}^{2} \mathbf{p}}{\mathbf{E}}\right\rangle_{+}=\left\langle\mathbf{V}_{\mathbf{g p}}\right\rangle_{+}
$$


where \langle\rangle$_{+}$denotes expectation value with respect to a positive-energy packet. Clearly (10) shows that the velocity is luminal. Indeed Dirac himself noticed this as soon as he had derived the relativistic electron equation [15] and rationalized that all measurements are averages over a short interval of time of the order of the Compton time, in which the Zitterbewegung effects are smoothened out and we get a sub luminal value for the velocity. To put it another way, a wave packet constructed of solutions of one sign of energy alone move with luminal velocities. We need a wave packet consisting of both positive and negative energies to get the usual sub luminal velocities and mass. This has been commented upon [16].

Turning to the neutrino we can now describe the neutrino as being made up of one sign of energy solutions alone, leading to its luminal speeds and near vanishing mass. This also means that Dirac spinor equation which consists of four components, two representing positive solutions and two representing negative solutions becomes a two component, that is positive only or negative only spinor. This of course is the well known Weyl equation which represents neutrinos as two component objects. All this explains several features including the near masslessness and the chirality.

In any case it can be argued that a particle localized in space would be described by a packet that contains both positive energy and negative energy solutions. Those particles described by only positive or only negative energy solutions would be luminal and therefore massless. Interestingly this would also mean that the Compton wavelength of such particles, in this case neutrinos would be infinite (or in practise very large) [14, 17, 18, 16].

Interestingly the cosmic neutrino background has recently been used by the author to explain the cosmological constant [19]. The starting point is the cold Fermi degenerate gas for which we have

$$
p_{F}^{3}=\hbar^{3}(N / V)
$$

where we can deduce this by using the fact that the ground state of such a Fermi assembly is such that the neutrinos occupy the lowest possible energy levels, while all the levels up to the Fermi energy $\epsilon$ or $e_{F}$ are filled up. That is these neutrinos fill the sphere of radius $p_{F}$. So we have

$$
\frac{V}{h^{3}} \int_{e_{p}<e_{F}} d^{3} p=N
$$

Remembering that we have,

$$
e_{F} \sim p_{F}^{2} / m
$$


(11) follows.

Feeding in the known neutrino parameter, viz., [20] $N \sim 10^{90}$ we get from the above, the consistent neutrino mass $\sim 10^{-3} \mathrm{eV}[21]$ and the background temperature $T \sim 1^{\circ} K$ as $K T$ is nothing but the Fermi energy $e_{F}$. More recently there has been hope that neutrinos can also exhibit the ripples of the early Big Bang and in fact, Trotta and Melchiorri claim to have done so [22].

It may be mentioned that there is growing evidence for the cosmic background neutrinos [1]. The GZK photo pion process seems to be the contributing factor.

Next using the expression for the Fermi energy

$$
\text { Fermi Energy }=\frac{N^{5 / 3} \hbar^{2}}{m_{\nu} R^{2}}=M \Lambda R^{2}
$$

where $M$ is the mass of the universe, $R$ its radius $\sim 10^{27} \mathrm{~cm}$, and $\Lambda$ is the cosmological constant.

We now get from (13),

$$
\Lambda \sim 10^{-37} \sec ^{-2}
$$

(14) describes the correct order of the cosmological constant.

It may be noted that by conventional arguments we get a wrong value of the cosmological constant that is $10^{120}$ orders of magnitude of the actually observed cosmological constant.

\section{References}

[1] Weiler, T.J. (2005). Int.J.Mod.Phys.A 20, (6), pp.1168-1179.

[2] Misner, C.W., Thorne, K.S. and Wheeler, J.A. (1973). Gravitation (W.H. Freeman, San Francisco), pp.819ff.

[3] Sidharth, B.G. (1999). Journal of Statistical Physics 95(3/4), May 1999.

[4] Sidharth, B.G. (1995). CAMCS TR 1995.

[5] Geim, A.K. (2009) Graphe: Status and Prospects in Science Vol.324, pp.1530-1534.

[6] Castro Neto, A.H., Guinea, F., Peres, N.M.R., Novoselov, K.S. and Geim, A.K. (2009). Reviews of Modern Physics Vol.81, pp.109-162. 
[7] Novoselov, K.S. et al. (2005). Two-dimensional gas of massless Dirac fermions in graphene in Nature Vol.438, pp.197-200.

[8] S.S. Schweber. (1961). An Introduction to Relativistic Quantum Field Theory (Harper and Row, New York).

[9] Huang, K. (1975). Statistical Mechanics (Wiley Eastern, New Delhi), pp.75ff.

[10] Sidharth, B.G. (2001). Chaos, Solitons and Fractals 12, pp.1563-1564.

[11] Witten, E. (1998). Advances in Theoretical and Mathematical Physics Vol.2, 1998, pp.253-291.

[12] Sidharth, B.G. (2008). The Thermodynamic Universe (World Scientific, Singapore).

[13] Sidharth, B.G. and Popova, A.D. (1997). Nonlinear World pp.4.

[14] Bjorken, J.D. and Drell, S.D. (1964). Relativistic Quantum Mechanics Vol.1 (Mc-Graw Hill Science Publishing, New York), pp.36.

[15] Dirac, P.A.M. (1958). The Principles of Quantum Mechanics (Clarendon Press, Oxford), pp.4ff, pp.253ff.

[16] Sidharth, B.G. (2011). New Advances in Physics Vol. 5(2). 2011,pp.5566.

[17] Feshbach, H. and Villars, F. (1958). Reviews of Modern Physics Vol.30, No.1, January 1958, pp.24-45.

[18] Greiner, W., Muller, B. and Rafelski, J. (1985). Quantum Electrodynamics of Strong Fields (Springer-Verlag, Berlin).

[19] Sidharth, B.G. (2013). New Advances in Physics Vol. 7 (1) 2013.

[20] Fang, L.Z. and Ruffini, R. (1984). Cosmology of the Early Universe (World Scientific, Singapore, 1984) p.93.

[21] Tegmark, M. et al. (2004). Phys.Rev.D. 69, 103501.

[22] Trotta, R., Melchiorri, A. www.astro.physics.ox.ac.uk/rxt/pdf/CNB. 